\title{
The ClpS-like N-domain is essential for the functioning of Ubr11, an N-recognin in Schizosaccharomyces pombe
}

\author{
Kenji Kitamura
}

\begin{abstract}
Several Ubr ubiquitin ligases recognize the $\mathrm{N}$-terminal amino acid of substrate proteins and promote their degradation via the Arg/N-end rule pathway. The primary destabilizing $\mathrm{N}$-terminal amino acids in yeast are classified into type 1 (Arg, Lys, and His) and type 2 (Phe, Trp, Tyr, Leu, lle, and Met- $\Phi$ ) residues. The type 1 and type 2 residues bind to the UBR box and the ClpS/N-domain, respectively, in canonical Ubr ubiquitin ligases that act as N-recognins. In this study, the requirement for type 1 and type 2 amino acid recognition by Schizosaccharomyces pombe Ubr11 was examined in vivo. Consistent with the results of previous studies, the $u b r 11 \Delta$ null mutant was found to be defective in oligopeptide uptake and resistant to ergosterol synthesis inhibitors. Furthermore, the ubr11 $\Delta$ mutant was also less sensitive to some protein synthesis inhibitors. A ubr11 ClpS/N-domain mutant, which retained ubiquitin ligase activity but could not recognize type 2 amino acids, phenocopied all known defects of the ubr11 1 mutant. However, the recognition of type 1 residues by Ubr11 was not required for its functioning, and no severe physiological abnormalities were observed in a ubr11 mutant defective in the recognition of type 1 residues. These results reinforce the fundamental importance of the ClpS/N-domain for the functioning of the N-recognin, Ubr11.
\end{abstract}

Keywords: Ubr protein; Ubiquitin ligase; N-end rule pathway; N-recognin; Oligopeptide transporter; Yeast

\section{Background}

The N-end rule pathway is responsible for the ubiquitination of substrate proteins that harbor a destabilizing amino acid at their $\mathrm{N}$-terminus. The destabilizing amino acid is an essential component of a degradation signal known as the $\mathrm{N}$-degron. A Ubr ubiquitin ligase, acting as an $\mathrm{N}$-recognin, binds to the $\mathrm{N}$-terminal residue of the $\mathrm{N}$-degron and ubiquitinates the substrate protein, thus targeting it for degradation by the proteasome (Tasaki et al. 2012; Varshavsky 2011). Two independent pathways, the Arg/N-end rule and the acetylated $\mathrm{N}$-end rule, have been described for this process. Ubr ubiquitin ligases function only in the Arg/N-end rule pathway.

Arg/N-end rule N-recognin mutants of the yeasts, Saccharomyces cerevisiae (ubr1) and Schizosaccharomyces pombe (ubr11), grow almost normally. However, because the Ubr proteins are required for the expression of oligopeptide transporters, both mutants are defective

Correspondence: kkita@hiroshima-u.ac.jp

Center for Gene Science, Hiroshima University, 1-4-2 Kagamiyama, Higashi-Hiroshima 739-8527, Japan in the uptake of extracellular oligopeptides (Byrd et al. 1998; Kitamura et al. 2012). The regulation of peptide uptake has been well characterized in S. cerevisiae (Turner et al. 2000; Du et al. 2002; Xia et al. 2008a, b; Varshavsky 2011). After Ubr1 ubiquitinates and promotes the degradation of the transcriptional repressor, Cup9, the expression of the peptide transporter gene (PTR2) is upregulated, and the cell becomes competent for peptide uptake. The binding of the intracellular N-end rule dipeptides to Ubr1 facilitates the recognition and ubiquitination of Cup9 by Ubr1, and further accelerates peptide uptake. Similarly, Ubr11 in S. pombe is essential for the expression of the oligopeptide transporter genes, ptr2 and isp4 (Kitamura et al. 2012). However, no Cup9 homolog has been found in the S. pombe genome, and the Ubr11 substrate that represses peptide uptake in S. pombe has not been identified. In the opportunistic pathogen, Candida albicans, Ubr1 controls hyphal initiation through the proteolysis of Cup9. However, it is not known whether the degradation of Cup9 depends on the type of residue present at its N-terminus (Lu et al. 2014). Similarly, the 
Ubr1-related protein, Ubl1, in the plant pathogen Fusarium is necessary for virulence against wheat and maize; however, the relevant substrate proteins are unknown (Ridenour et al. 2013).

The classical primary destabilizing amino acid in the $\mathrm{N}$-degrons of the Arg/N-end rule pathway in yeast is either a basic residue (type 1: Arg, Lys, or His) or a bulky hydrophobic residue (type 2: Leu, Ile, Trp, Phe, or Tyr) (Varshavsky 2011; Tasaki et al. 2012). An unacetylated $\mathrm{N}$-terminal methionine followed by a hydrophobic residue (designated Met- $\Phi$ also functions as a type $2 \mathrm{~N}$-degron (Kim et al. 2014). Different regions within the canonical Ubr $\mathrm{N}$-recognin proteins bind to type 1 and type 2 amino acids (Xia et al. 2008b; Tasaki et al. 2009). Type $2 \mathrm{~N}$-terminal amino acids bind to the $\mathrm{N}$-domain, which is homologous to the bacterial N-recognin, ClpS (Lupas and Koretke 2003; Erbse et al. 2006; Tasaki et al. 2009). Similar to the eukaryotic $\mathrm{N}$-end rule pathway, $\mathrm{ClpS}$ is responsible for the recognition of the same $\mathrm{N}$-terminal type 2 amino acids (except Ile) in the bacterial $\mathrm{N}$-end rule pathway (Erbse et al. 2006; Dougan et al. 2010). In contrast, type $1 \mathrm{~N}$-terminal residues are recognized by the eukaryotespecific UBR domain. In our previous studies, we investigated the roles of these domains and demonstrated the importance of type $2 \mathrm{~N}$-terminal amino acid recognition in peptide uptake, by mutating Ubr11 in $S$. pombe (Kitamura and Fujiwara 2013). However, how the recognition of type 1 residues affects the in vivo function of Ubr11 was not characterized.

In the present study, a Ubr11 mutant that was defective only in the recognition of type $1 \mathrm{~N}$-terminal amino acids was engineered, and the effects of the mutation were compared with those in a Ubr11 ClpS/N-domain mutant defective in the recognition of type 2 residues. Importantly, it was found that the recognition of type 2 residues by the $\mathrm{ClpS} / \mathrm{N}$-domain was essential, but the recognition of type 1 residues by the UBR domain was dispensable for almost all Ubr11 functions. These results contribute to our understanding of the structure-function relationship in canonical Ubr ubiquitin ligases.

\section{Results}

\section{Analysis of a ubr11 mutant defective in type 1 amino acid recognition}

The residues of bacterial $\mathrm{ClpS}$ that interact with the hydrophobic N-degrons were identified in earlier studies (Wang et al. 2008; Román-Hernández et al. 2009; Schuenemann et al. 2009). We previously characterized a yeast strain that harbored a mutation within the conserved ClpS/N-domain in S. pombe Ubr11. This ubr11-m3 mutant (D251N, H254Y), which was renamed ubr11-T2 in this study (Additional file 1: Figure S1) because of its 'type 2-defective' nature, lacked dipeptide uptake because of its inability to express the Ptr2 peptide transporter
(Kitamura and Fujiwara 2013). In the ubr11-T2 mutant, which was unable to recognize type 2 residues, the fluorescence intensity of the type 1 model substrate, $\mathrm{Arg}^{\mathrm{Nd}}$-fused green fluorescent protein $\left(\mathrm{Arg}^{\mathrm{Nd}}\right.$-GFP), was low, similar to that in wild type Ubr11-expressing cells (control profiles in Figure 1a). We previously demonstrated that the fluorescence intensity reflects the protein amount of an $\mathrm{N}$-degron, $\mathrm{Xaa}^{\mathrm{Nd}}$ bearing-GFP, and that a low GFP level correlates with an intrinsic instability of GFP, because of its $\mathrm{N}$-end rule-dependent proteolysis (Kitamura and Fujiwara 2013). Because the $\mathrm{N}$-end rule substrate and the $\mathrm{N}$-end rule dipeptide compete for the same binding site within Ubr11, proteolysis via the $\mathrm{N}$-end rule pathway is inhibited by dipeptides that bear the same type of N-terminal amino acid as the substrate. In contrast to the wild type Ubr11-expressing cells, degradation was not inhibited by exogenous Lys-Leu type 1 dipeptides in the ubr11-T2 mutant (Figure 1a, vector) because it was defective in the expression of the Ptr2 dipeptide transporter (Kitamura and Fujiwara 2013). However, when a multicopy plasmid was used to increase the expression of Ubr11-T2, Lys-Leu dipeptides weakly inhibited the degradation of Arg $^{\text {Nd }}$-GFP, although not as effectively as in a strain expressing wild type Ubr11 (Figure 1a, right, and b; Additional file 2: Figure S2b). Ubr11's recognition of Lys-Leu is not affected by the ubr11-T2 mutation itself (Kitamura and Fujiwara 2013). Therefore, these results indicated that Ubr11-T2 was not completely inactive, but its ability to induce peptide uptake was severely compromised.

To complement the above findings and to further investigate the relationship between $\mathrm{N}$-terminal amino acid recognition and peptide uptake, a Ubr11 mutant defective in the recognition of type 1 residues was characterized. The ubr11-m6 mutant (Asp117Ala; Additional file 1: Figure S1) was tested first because the conserved aspartic acid interacts with the type $1 \mathrm{~N}$-terminal amino acid of substrate proteins (Choi et al. 2010; Matta-Camacho et al. 2010). Further, a corresponding mutation in mouse Ubr1 specifically interferes with its ability to bind type 1 amino acids in vitro (Tasaki et al. 2009). However, its functionality in vivo was not tested. The mutant Ubr11-m6 protein was expressed in the $u b r 11 \Delta$ strain from a multicopy plasmid, and its functionality was monitored. Interestingly, the $S$. pombe ubr11-m6 mutant was able to degrade both type 1 and type 2 model substrates (Additional file 2: Figure S2a and b) and induce peptide uptake (Figure 2). Another mutant, ubr11-T1 (Gly147Arg), was then examined because a mutation of the corresponding residue in S. cerevisiae Ubr1 produced a mutant that was defective in targeting type 1 substrates (Xia et al. 2008b). This conserved glycine is also located near the residue critical for type $1 \mathrm{~N}$-end recognition (Additional file 1: Figure S1). The type 1 model substrate, $\mathrm{Arg}^{\mathrm{Nd}}$-GFP, was highly expressed in the S. pombe 


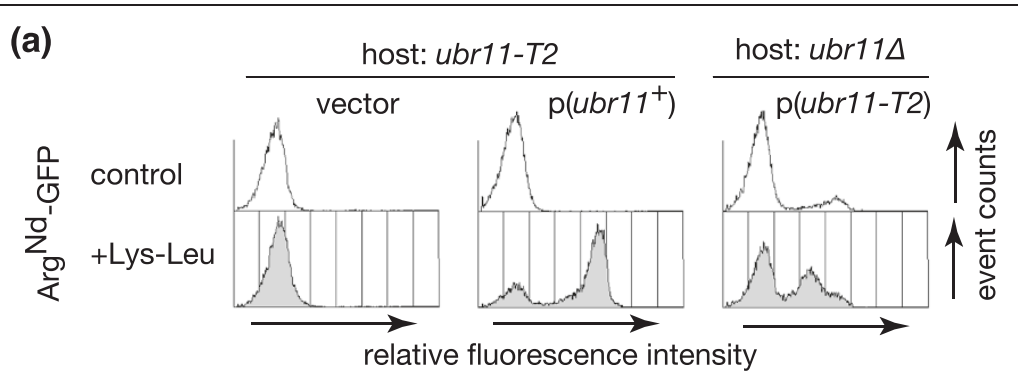

(b)

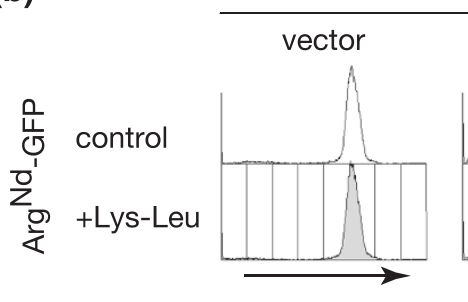

host: ubr11

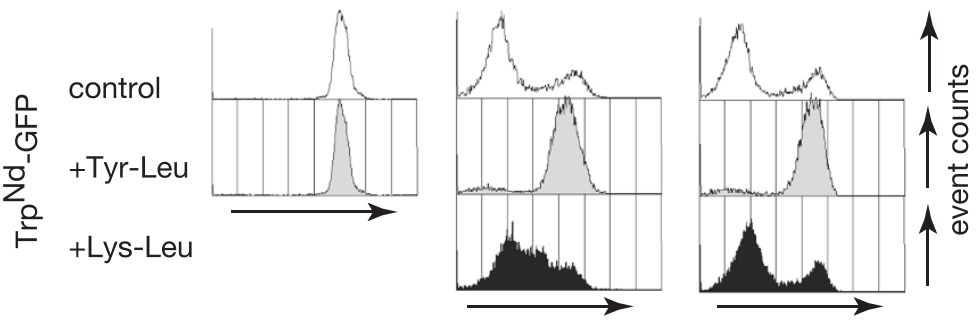

relative fluorescence intensity

(c)

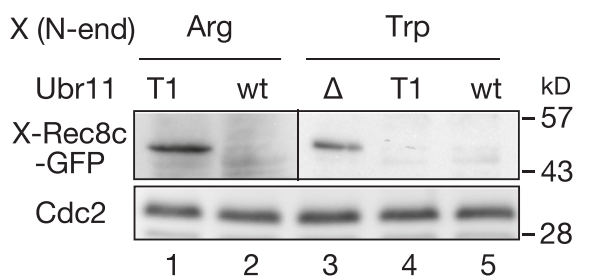

(d) Arg-Rec8c: RVQGELGLNIPWNTSSRSNSAINSK .....

Trp-Rec8c: WVQGELGLNIPWNTSSRSNSAINSK ....

ArgNd: $\quad$ RHKSGAWKLPVSLVK (DYKDDDDK)

TrPNd: WHKSGAWKLPVSLVK (DYKDDDDK)

Figure 1 Characterization of $u b r 11$ mutants specifically defective in the recognition of $\mathrm{N}$-terminal type 1 or type 2 residues. (a and $\mathrm{b}$ ) Each strain was cultured with or without the indicated dipeptide for $4 \mathrm{~h}$. The relative fluorescence intensities of Arg $^{\text {Nd_-GFP and Trp }}{ }^{\text {Nd }}$-GFP, which are type 1 and type $2 \mathrm{~N}$-end rule substrates, respectively, were measured by flow cytometry. (a) The effect of Lys-Leu dipeptides was severely compromised in the ubr11-T2 mutant. ubr11-T2, which has type $2 \mathrm{~N}$-terminal residue-specific recognition defect (left and middle), or the ubr11 $\Delta$ (right) strains, which express Arg ${ }^{\mathrm{Nd}}$-GFP, were transformed with the indicated plasmid, and monitored for changes in the fluorescence intensity of

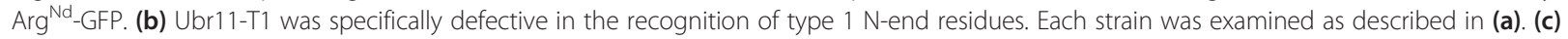
The type $1 \mathrm{~N}$-degron sequence in Rec8 did not promote Rec8c degradation in the ubr11-T1 strain. The X-Rec8c-GFP was expressed from the $n m t$ promoter. The steady state levels of the type 1 substrate, Arg-Rec8c-GFP (lanes 1 and 2), and the type 2 substrate, Trp-Rec8c-GFP (lanes 3-5), in the indicated strains were examined by immunoblotting with anti-GFP antibody. Cdc2: loading control. (d) Amino acid sequences of the $\mathrm{N}$-degrons used in this study. The DYKDDDDK sequence shown in parenthesis is a FLAG tag epitope that was inserted before the GFP protein.

ubr11-T1 mutant (Figure 1b, top right panel; Additional file 2: Figure $\mathrm{S} 2 \mathrm{~b}$ ), as in the control $u b r 11 \Delta$ strain (Figure 1b, top left panel, vector; Additional file 2: Figure S2b). In contrast, the fluorescence intensity of the type 2 substrate, $\operatorname{Trp}^{\mathrm{Nd}}$-GFP, was low and its level increased when the type 2 dipeptide, Tyr-Leu, was added to strains expressing either 
wild type Ubr11 or Ubr11-T1. Therefore, the ubiquitin ligase activity of Ubr11-T1 was not impaired, and the ubr11-T1 mutant had a specific defect in type 1 residue recognition, but responded normally to the type 2 dipeptide. For reasons that are still unclear, the fluorescence intensity and protein levels of the type 2 substrate, $\operatorname{Trp}^{\mathrm{Nd}}$-GFP, were partially increased when type 1 Lys-Leu dipeptides were added to a strain expressing wild type Ubr11 (Figure 1b, left profile shown in black; Additional file 3: Figure S3a and b). However, Lys-Leu dipeptides were not effective in the ubr11-T1 mutant (Figure 1b), confirming that this mutant had lost the ability to interact with type $1 \mathrm{~N}$-terminal amino acids.

To exclude the possibility that the defect in the $u b r 11-T 1$ mutant was confined to the $\mathrm{Arg}^{\mathrm{Nd}} \mathrm{N}$-degron, the stability of another $\mathrm{N}$-end rule substrate, bearing a different $\mathrm{N}$-degron, was examined. The $\mathrm{C}$-terminal fragment of Rec8 (Rec8c), which is generated by a separase-mediated cleavage of cohesin, is an endogenous substrate of the Arg/N-end rule pathway in S. pombe (Fujiwara et al. 2013). Although the $\mathrm{N}$-degron sequence in Arg-Rec8c is completely different from that in $\mathrm{Arg}^{\mathrm{Nd}}$ (Figure 1d), Arg-Rec8c-GFP (type 1 substrate), but not Trp-Rec8c-GFP (type 2 substrate), was stabilized in the $u b r 11-T 1$ mutant (Figure 1c). Therefore, it was concluded that the ubr11-T1 mutant was defective in the recognition of $\mathrm{N}$-terminal type 1 amino acids in general.

\section{Utilization of dipeptides by ubr11 mutants defective in recognizing type 1 or type 2 residues}

Subsequently, the ability of the mutants to support peptide uptake was tested. All proteins were expressed in the ubr $11 \Delta$ host strain from a multicopy plasmid. Consistent with our previous report (Kitamura et al. 2012), ubr11 cells harboring the empty vector were defective in the uptake of all dipeptides examined (Figure 2). Interestingly, the uptake of all three dipeptides was not affected in the
ubr11-T1 mutant. However, the ubr11-T2 mutant plasmid failed to rescue the growth when type 2 (Leu-Ala) or non-N-end rule (Ala-Leu) dipeptides were used. Growth could not be recovered even when soy peptides (various soy-derived oligopeptides-enriched mixtures; Kitamura et al. 2012) were used as a source of leucine. Although a ubr11s strain harboring the multicopy ubr11-T2 plasmid was able to utilize type 1 dipeptides (Lys-Leu), a mutant strain, in which the genomic $u b r 11^{+}$ locus was replaced with the $u b r 11-T 2$ gene, failed to utilize Lys-Leu (Kitamura and Fujiwara 2013). In conclusion, Ubr11 stimulated dipeptide uptake even when it was unable to recognize $\mathrm{N}$-terminal type 1 amino acids. In contrast, recognition of type 2 amino acids had a pivotal role in peptide uptake.

Resistance of $u b r 11$ mutants to protein synthesis inhibitors When characterizing the $u b r$ mutants, it was observed that the $u b r 11 \Delta$ mutant showed weak resistance towards low doses of protein synthesis inhibitors, such as anisomycin and hygromycin B (Figure 3a). The expression of two major oligopeptide transporters, Ptr2 and Isp4, is very low in ubr11 cells (Kitamura et al. 2012). However, like the wild type strain, the ptr $2 \Delta$ isp $4 \Delta$ double mutant was sensitive to both inhibitors, indicating that the $u b r 11 \Delta$ mutant was inherently less sensitive to inhibition of peptide synthesis, and that Ptr2 and Isp4 were unrelated to the resistance. Next, the effect of type 1 and type 2 recognition defects on the cells' sensitivity to protein synthesis inhibitors was tested. Only the ubr11-T2 mutant, but not ubr11-T1, was resistant to anisomycin (Figure $3 \mathrm{~b})$. Both the $u b r 11-T 1$ and the $u b r 11-T 2$ mutants were resistant to hygromycin B at $40 \mu \mathrm{g} / \mathrm{mL}$, though the effect on Ubr11-T2 was more pronounced. These results suggested that an impairment in the functionality of the ClpS/N-domain affected the sensitivity towards these protein synthesis inhibitors. 


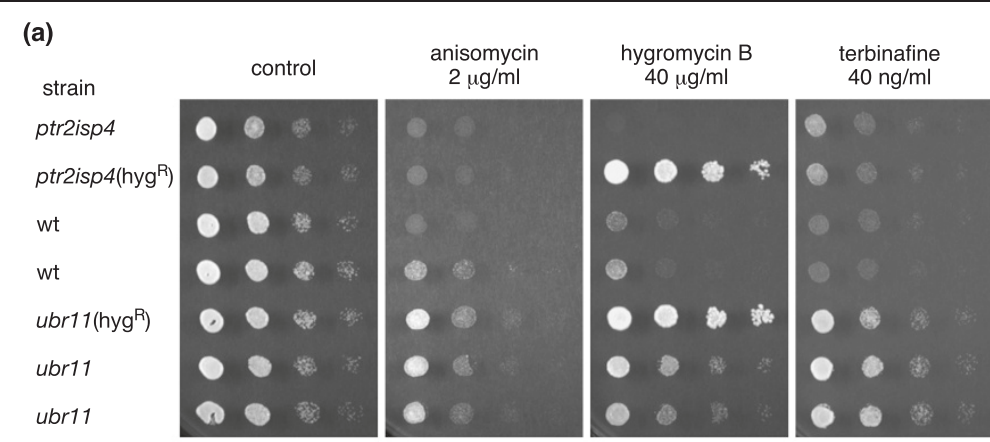

(b)
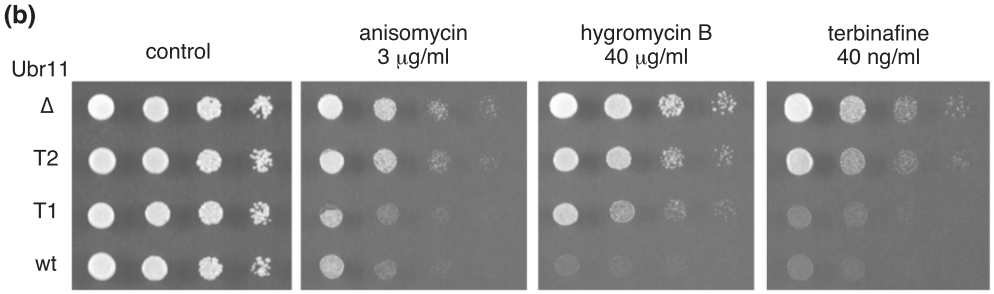

Figure 3 The ubr11-T2 mutant phenocopied ubr11 mutant for drug resistance. (a) Resistance of the ubr11 $\Delta$ mutant to anisomycin and hygromycin B (inhibitors of protein synthesis) and terbinafine (an inhibitor of ergosterol synthesis). Serially diluted cells of each strain were spotted on drug-containing or drug-free (control) medium. Note that the strains shown in the second and fifth rows are intrinsically resistant to hygromycin B because they contain the isp4::hphMX6 or ubr17::hphMX6 allele. (b) The host ubr11 $\triangle$ strain was transformed with plasmids encoding wild type or mutant Ubr11, and the sensitivity of the yeast to the inhibitors was examined as in (a). The ubr11 $\Delta$ and ubr11-T2 strains showed the same profiles, but the ubr11-T1 profile resembled that of the wild type strain.

\section{Mutation in the ubr11 ClpS/N-domain mitigated the growth inhibitory effects of terbinafine}

A $u b r 11 \Delta$ mutant was identified in a genome-wide screen for mutants that were resistant to terbinafine and clotrimazole, both of which inhibit different steps of the ergosterol synthetic pathway (Fang et al. 2012). Although the reason for the resistance remains unclear, the resistance to terbinafine was confirmed using an independently prepared ubr11 $\Delta$ strain; it was established that both Ptr2 and Isp4 were not responsible for the resistance (Figure 3a). Interestingly, the ubr11-T2 ClpS/N-domain mutant was also resistant to terbinafine, whereas cells expressing Ubr11-T1 or the wild type protein were sensitive (Figure $3 \mathrm{~b}$ ), suggesting that the integrity of the $\mathrm{ClpS} / \mathrm{N}$-domain is also important for the response towards terbinafine.

\section{Discussion}

In this study, the relationship between the recognition of $\mathrm{N}$-terminal residues in the Arg/N-end rule pathway and the in vivo function of the N-recognin, Ubr11, in S. pombe was examined. The Arg/N-end rule pathway in yeast plays an important role in the regulation of extracellular oligopeptide uptake by promoting the expression of peptide transporters (Varshavsky 2011; Kitamura et al. 2012). In $S$. cerevisiae, the Ubr1-dependent proteolysis of the transcriptional repressor, Cup9, by the Arg/N-end rule pathway is essential for peptide uptake (Turner et al. 2000; Du et al. 2002; Xia et al. 2008a). Curiously, Cup9 is neither a type 1 nor a type $2 \mathrm{~N}$-end rule substrate
(Xia et al. 2008b). The binding of $\mathrm{N}$-end rule dipeptides to the N-recognin, Ubr1, increases the degradation of Cup9, thus further stimulating peptide uptake (Turner et al. 2000). Interestingly, the S. pombe genome does not encode a Cup9 homolog. In this study, in S. pombe, the recognition of $\mathrm{N}$-terminal type 1 amino acids was found to be dispensable, whereas the recognition of type 2 amino acids was found to be critical for the in vivo function of Ubr11. Based on the results of this study, we can assume that the $\mathrm{N}$-end rule substrate required for peptide uptake in $S$. pombe may be a type 2 substrate protein. However, the identity of this protein remains unknown. Alternatively, the $\mathrm{ClpS} / \mathrm{N}$-domain of Ubr11 may regulate peptide uptake by recognizing an intracellular type 2 oligopeptide itself, rather than a type 2 substrate protein.

To date, several regulators of cellular physiology, including transcription factors that regulate hypoxic responses, caspase-generated pro-apoptotic protein fragments, neurodegeneration-associated protein fragments, proteolytic fragment of BRCA1 (breast cancer susceptibility type 1 protein), and PINK1 (a Parkinson disease-related mitochondrial protein) have been identified as substrates of the Arg/N-end rule pathway in plants and mammals (Gibbs et al. 2011; Licausi et al. 2011; Piatkov et al. 2012; $\mathrm{Xu}$ et al. 2012; Brower et al. 2013; Yamamoto and Youle 2013). However, these proteins are not conserved in yeast. In $S$. cerevisiae and $S$. pombe, other than the type 2 Met- $\Phi$ degron-bearing proteins, whose N-termini are inherently acetylated (Kim et al. 2014), only the C-terminal fragments 
of the mitotic cohesin subunit, Scc1, and its meiotic counterpart, $\operatorname{Rec} 8$, have been identified as Arg/N-end rule substrates whose degradation depends on the Nterminal residue (Rao et al. 2001; Fujiwara et al. 2013). The C-terminal fragments of Scc1 and Rec8, generated by a separase-mediated cleavage during mitosis and meiosis, respectively, bear a type $1 \mathrm{~N}$-end residue. Inhibiting the degradation of the Scc1 and Rec8 fragments is not deleterious unless the proteins are strongly overexpressed from an ectopic promoter (Rao et al. 2001; Fujiwara et al. 2013). Taken together with our finding that a ubr11-T1 mutation does not result in any apparent defects and that the ubr11 1 mutant has no adverse phenotypes in meiosis (Fujiwara et al. 2013), these observations suggest that there may be no essential type $1 \mathrm{~N}$-end rule substrates that need to be degraded in $S$. pombe, at least in an unperturbed condition. It is still possible that the degradation of an unidentified type 1 substrate is required for survival in a specific condition. However, such drugs or conditions, under which the $u b r 11$ mutation results in deleterious effects in $S$. pombe, have not been encountered. Apparently, the degradation of type 1 substrates by the Arg/N-end rule pathway may have gained significance in higher eukaryotes during the course of evolution.

Type $1 \mathrm{~N}$-degrons appear to induce proteolysis more potently than type $2 \mathrm{~N}$-degrons in S. pombe (Kitamura and Fujiwara 2013; Fujiwara et al. 2013). However, the recognition of type 2 amino acids seems to play a more important role than the recognition of type 1 residues, as demonstrated in this study. Intriguingly, among the canonical Ubr proteins, the ClpS/N-domain, which recognizes type 2 amino acids, is conserved only in genuine $\mathrm{N}$-recognins (Tasaki et al. 2012). The $\mathrm{N}$-domain sequence in Ubr proteins was originally identified on the basis of its homology to a small bacterial N-recognin protein, ClpS (Lupas and Koretke 2003; Erbse et al. 2006; Tasaki et al. 2009). By incorporating the ClpS-like N-domain, canonical Ubr N-recognins in eukaryotes acquired a specialized role for the recognition of $\mathrm{N}$-end residue in the Arg/N-end rule pathway and, simultaneously, a means to regulate peptide uptake. The binding region for type 1 residues is found within the eukaryote-specific UBR domain that characterizes all Ubr proteins, including the non-N-recognins and the non-canonical Ubr proteins (mammalian Ubr3-Ubr7, S. pombe Ubr1, and S. cerevisiae Ubr2) (Varshavsky 2011; Tasaki et al. 2012). Previous studies have shown that the degradation of type $2 \mathrm{~N}$-end rule substrates is stimulated in the presence of type 1 dipeptides (Reiss et al. 1988; Gonda et al. 1989; Baker and Varshavsky 1991; Kwon et al. 2001). Considering this, an unexpected observation in this study was that exogenous type $1 \mathrm{di}$ peptides increased the amount of type 2 substrate. In the case of $\operatorname{Trp}^{\mathrm{Nd}}$-GFP, an increase was observed for at least two different type 1 dipeptides, Lys-Leu and Arg-Phe
(Figure 1b, Additional file 3: Figure S3). In contrast, the type 2 dipeptide, Tyr-Leu, did not affect the levels of $\mathrm{Arg}^{\mathrm{Nd}}$-GFP, a type 1 substrate (Kitamura and Fujiwara 2013). Although the molecular mechanisms underlying this phenomenon need to be elucidated in future studies, the current data indicate that the recognition of type 2 substrates by $\mathrm{N}$-recognins is strongly influenced by the presence of type 1 dipeptides in a complex manner.

Ubr ubiquitin ligases are necessary for protein quality control (Eisele and Wolf 2008; Heck et al. 2010; Nillegoda et al. 2010; Prasad et al. 2010; Khosrow-Khavar et al. 2012; Theodoraki et al. 2012: Stolz et al. 2013; Summers et al. 2013; Kriegenburg et al. 2014). In both S. cerevisiae and $S$. pombe, a mutation in the Ubr N-recognin alleviates growth defects in several temperature-sensitive mutants (Khosrow-Khavar et al. 2012; Kriegenburg et al. 2014). In this study, it was found that the $u b r 11 \Delta$ mutant was less sensitive to protein synthesis inhibitors than the wild type strains, suggesting that Ubr11 also contributes to quality control when partially defective proteins that retain some residual function are produced in the presence of low concentrations of protein synthesis inhibitors. Alternatively, substrates of Ubr11 may be required for certain critical cellular processes that are more sensitive to protein level alterations. In either case, inactivation of the Ubr11-dependent proteolysis of substrates alleviates growth inhibition. Interestingly, in S. cerevisiae, the quality control of several proteins is mediated by the Arg/N-end rule pathway, in which an unacetylated $\mathrm{N}$-terminal methionine is recognized by the type 2 amino acid-binding site within Ubr1 (Kim et al. 2014). However, other studies have shown that the role of the Ubr proteins in quality control is independent of the N-end rule pathway (Heck et al. 2010; Nillegoda et al. 2010). Whether the Ubr proteins function as N-recognins or act independently of the $\mathrm{N}$-end rule pathway may depend on the substrate protein. As shown in this study, the ubr11 ClpS/N-domain mutant displayed altered responses to several drugs. Whether these defects involve recognition of substrates with type $2 \mathrm{~N}$-end residues remains to be determined.

\section{Conclusions}

Ubr11 N-recognin in Schizosaccharomyces pombe is a conserved ubiquitin ligase, which is essential for the degradation of substrate proteins harboring destabilizing $\mathrm{N}$-terminal amino acids (N-degron) via the Arg/N-end rule pathway. The N-domain in Ubr11, which is homologous to the bacterial ClpS, recognizes bulky hydrophobic amino acids at the $\mathrm{N}$-terminus (type $2 \mathrm{~N}$-degron). This report described a $\mathrm{ClpS} / \mathrm{N}$-domain mutant, which did not recognize type $2 \mathrm{~N}$-end residues but retained ubiquitin ligase activity towards type 1 substrates, phenocopied the ubr11 $\Delta$ null mutant. Specifically, the ClpS/N-domain 
mutant was resistant to inhibitors of ergosterol synthesis (terbinafine) and protein synthesis (hygromycin B and anisomycin). These findings indicate that the $\mathrm{ClpS} / \mathrm{N}$-domain has a general role in all cellular functions of the Ubr11 protein, in addition to its known role as a recognition site for $\mathrm{N}$-degron.

\section{Materials and methods}

\section{Yeast strains and culture conditions}

The yeast strains used in this study are listed in Additional file 4: Table S1. Rich complete medium (YES) and synthetic minimal medium (EMM2) were used for cell culture. These media and other general yeast methods have been described previously (Forsburg and Rhind 2006). Ammonium chloride (the nitrogen source in EMM2) was replaced with sodium glutamate $(0.38 \% \mathrm{w} / \mathrm{v})$ to evaluate the sensitivity of yeast to anisomycin, hygromycin $\mathrm{B}$, and terbinafine. Dipeptides were purchased from Sigma-Aldrich Japan (Tokyo, Japan), Bachem (Bubendorf, Switzerland), and Kokusan Chemical Co. Ltd. (Tokyo, Japan), and used at $0.2 \mathrm{mM}$ (to support cell growth) or $5 \mathrm{mM}$ (to inhibit proteolysis). Hi-Nute HK soy peptides (provided by Ms. Kitagawa, Fuji Oil, Osaka, Japan) were used at a concentration of $0.1 \%(\mathrm{w} / \mathrm{v})$.

To monitor proteolysis via the Arg/N-end rule pathway, the GFP-tagged model substrates, Xaa ${ }^{\mathrm{Nd}}$-GFP and X-Rec8c-GFP, were used as described previously (Fujiwara et al. 2013; Kitamura and Fujiwara 2013). To express these proteins from the $n m t$ promoter, the cells were grown in thiamine-free EMM2 for at least $18 \mathrm{~h}$ at $28^{\circ} \mathrm{C}$. To inhibit degradation via the Arg/N-end rule pathway, the cells were treated with dipeptides for 3-5 h.

\section{Plasmids}

The ubr11-m6 (Asp117Ala) and ubr11-T1 (Gly147Arg) mutants were synthesized as described previously by inverse polymerase chain reaction using the $\mathrm{Pk}-u b r 11^{+}$ template plasmid (Kitamura and Fujiwara 2013). After sequence verification, each $u b r 11$ gene, including the promoter region, was excised by PstI digestion and inserted into the PstI site of the pDblet vector (Brun et al. 1995). The ubr11-T2 mutant, which does not recognize type $2 \mathrm{~N}$-terminal residues, is identical to the ubr11-m3 mutant, which we reported in previous studies (Kitamura and Fujiwara 2013, Additional file 1: Figure S1). However, the mutant was renamed $u b r 11-T 2$ in this study, to emphasize its type- 2 residue-specific defect.

\section{Flow cytometry}

The relative fluorescence intensities of $\mathrm{Arg}^{\mathrm{Nd}}$-GFP and $\operatorname{Trp}^{\mathrm{Nd}}$-GFP were measured in 10000 live cells by flow cytometry using a FACSCalibur ${ }^{\mathrm{TM}}$ flow cytometer (Becton Dickinson, Franklin Lakes, NJ).

\section{Immunoblotting}

Total cellular protein extracts were prepared and used in immunoblotting experiments as described previously (Kitamura et al. 2011). Anti-GFP (GF200; Nacalai Tesque, Kyoto, Japan) and anti-Cdc2 (sc-53; Santa Cruz Biotechnology, Dallas, Texas) were used as the primary antibodies.

\section{Additional files}

\begin{abstract}
Additional file 1: Figure S1. Sequence alignments of Ubr boxes and ClpS/N-domains. Residues that are identical and homologous in at least three proteins are shown in red and blue, respectively. Highlighted in black in the S. pombe Ubr11 are the residues mutated in this study. The type 1 and type $2 \mathrm{~N}$-terminal amino acids of substrates or oligopeptides interact with the residues marked by asterisks in the Ubr box and ClpS/N-domain, respectively. Sp, Schizosaccharomyces pombe; Sc, Saccharomyces cerevisiae; Fv, Fusarium verticillioides; Ca, Candida albicans; Mm, Mus musculus; Ec, Escherichia coli; Cc, Caulobacter crescentus.
\end{abstract}

Additional file 2: Figure S2. Functionality of the ubr 11 mutants. (a) The ubr11-m6 mutant was functional. The ubr11 $\triangle$ strains which expressed Arg $^{\mathrm{Nd}}$-GFP (left) or Trp ${ }^{\mathrm{Nd}}$-GFP (right) were transformed with an autonomously replicating plasmid encoding the Ubr11-m6 protein, and analyzed by flow cytometry. The GFP fluorescence levels were low in untreated control cells due to proteolysis via the $\mathrm{N}$-end rule pathway. After $3 \mathrm{~h}$ of incubation in the presence of the dipeptides, the fluorescence intensities increased to a level similar to that in wild type Ubr11 expressing cells (Figure 1b). The peak to the right, with high fluorescence levels of $\mathrm{Trp}^{\mathrm{Nd}}$-GFP, in the control culture may represent the population that lost the plasmids because of their relatively unstable nature. (b) Functionality of the type 1- and type 2-specific ubr11 mutants. The Arg $^{\text {Nd }}$-GFP-expressing ubr $11 \triangle$ strain was transformed with the empty vector (lane 1, $\Delta$ ) or plasmids directing the expression of each Ubr11 mutant protein (lanes 2-10). The cells were cultured in the presence or absence of Lys-Leu dipeptides for $5 \mathrm{~h}$, and the GFP protein levels were compared by immunoblotting. The same extracts were independently examined for the ubr11-T2 mutant (lanes 3, 4, 9, and 10). Eight micrograms (lanes 1-8) and three micrograms (lanes 9 and 10) of total cell extracts were used. Cdc2: loading control.

Additional file 3: Figure S3. The type 1 dipeptides induced an increase in the Trp ${ }^{\mathrm{Nd}}$-GFP type 2 substrate protein levels. (a) Three wild type strains expressing Xaa ${ }^{\mathrm{Nd}}$-GFP from the same $n m t 1$ promoter were cultured for $5 \mathrm{~h}$ ( $\mathrm{Trp}^{\mathrm{Nd}}$-GFP and Met ${ }^{\mathrm{Nd}}$-GFP) or $3 \mathrm{~h}$ ( $\mathrm{Arg}^{\mathrm{Nd}}$-GFP) in the presence or absence of the dipeptides indicated. Relative fluorescence intensities in each culture were examined by flow cytometry. Unexpectedly, $\operatorname{Trp}^{\mathrm{Nd}}$-GFP fluorescence, in addition to $\mathrm{Arg}^{\mathrm{Nd}}$-GFP fluorescence, increased in response to two different type 1 dipeptides. In contrast, the levels of $\mathrm{Met}^{\mathrm{Nd}}$-GFP, which is not a substrate of the $\mathrm{N}$-end rule pathway, were not affected by the dipeptides. Only the $\mathrm{N}$-terminal amino acids (Arg, Trp, and Met) are different among these three GFP proteins, indicating that Lys-Leu did not generally affect the GFP levels. (b) Total cell extracts were prepared from the same culture that was analyzed in (a), and protein levels of Trp ${ }^{\mathrm{Nd}}$-GFP were examined by immunoblotting.

Additional file 4: Table S1. Schizosaccharomyces pombe strains used in this study.

\section{Competing interests}

The author has no competing interests.

\section{Authors' contributions}

KK designed the study, carried out the experiments, analyzed the data, and drafted the manuscript.

\section{Acknowledgments}

This work was supported in part by a Grant-in-Aid for Scientific Research on Priority Areas from the Ministry of Education, Culture, Sports, Science and 
Technology (MEXT), Japan. I thank Ichiro Yamashita and Nobukazu Tanaka for their help and discussions, and Tomoe Hikosaka for DNA sequencing.

\section{Received: 28 February 2014 Accepted: 16 May 2014}

\section{Published: 20 May 2014}

\section{References}

Baker RT, Varshavsky A (1991) Inhibition of the N-end rule pathway in living cells. Proc Natl Acad Sci U S A 88:1090-1094

Brower CS, Piatkov Kl, Varshavsky A (2013) Neurodegeneration-associated protein fragments as short-lived substrates of the N-end rule pathway. Mol Cell 50:161-171

Brun C, Dubey DD, Huberman JA (1995) pDblet, a stable autonomously replicating shuttle vector for Schizosaccharomyces pombe. Gene 164:173-177

Byrd C, Turner GC, Varshavsky A (1998) The N-end rule pathway controls the import of peptides through degradation of a transcriptional repressor. EMBO J 17:269-277

Choi WS, Jeong B-C, Joo YJ, Lee M-R, Kim J, Eck MJ, Song HK (2010) Structural basis for the recognition of $\mathrm{N}$-end rule substrates by the UBR box of ubiquitin ligases. Nat Struct Mol Biol 17:1175-1181

Dougan DA, Truscott KN, Zeth K (2010) The bacterial N-end rule pathway: expect the unexpected. Mol Microbiol 76:545-558

Du F, Navarro-Garcia F, Xia Z, Tasaki T, Varshavsky A (2002) Pairs of dipeptides synergistically activate the binding of substrate by ubiquitin ligase through dissociation of its auto inhibitory domain. Proc Natl Acad Sci U S A 99:14110-14115

Eisele F, Wolf DH (2008) Degradation of misfolded protein in the cytoplasm is mediated by the ubiquitin ligase Ubr1. FEBS Lett 582:4143-4146

Erbse A, Schmidt R, Bornemann T, Schneider-Mergener J, Mogk A, Zahn R, Dougan DA, Bukau B (2006) ClpS is an essential component of the $\mathrm{N}$-end rule pathway in Escherichia coli. Nature 439:753-756

Fang Y, Hu L, Zhou X, Jaiseng W, Zhang B, Takami T, Kuno T (2012) A genomewide srceen in Schizosaccharomyces pombe for genes affecting the sensitivity of antifungal drugs that target ergosterol biosynthesis. Antimicrob Agents Chemother 56:1949-1959

Forsburg SL, Rhind N (2006) Basic methods for fission yeast. Yeast 23:173-183

Fujiwara H, Tanaka N, Yamashita I, Kitamura K (2013) Essential role of Ubr11, not Ubr1 as an $\mathrm{N}$-end rule ubiquitin ligase in Schizosaccharomyces pombe. Yeast 30:1-11

Gibbs DJ, Lee SC, Isa NM, Gramuglia S, Fukao T, Bassel GW, Correia CS, Corbineau F, Theodoulou FL, Bailey-Serres J, Holdsworth MJ (2011) Homeostatic response to hypoxia is regulated by the $\mathrm{N}$-end rule pathway in plants. Nature 479:415-418

Gonda DK, Bachmair A, Wünning I, Tobias JW, Lane WS, Varshavsky A (1989) Universality and structure of the N-end rule. J Biol Chem 264:16700-16712

Heck JW, Cheung SK, Hampton RY (2010) Cytoplasmic protein quality control degradation mediated by parallel actions of the E3 ubiquitin ligases Ubr1 and San1. Proc Natl Acad Sci U S A 107:1106-1111

Khosrow-Khavar F, Fang NN, Ng AH, Winget JM, Comyn SA, Mayor T (2012) The yeast Ubr1 ubiqitin ligase participates in a prominent pathway that targets cytosolic themosensitive mutants for degradation. G3 (Bethesda) 2:619-628

Kim H-K, Kim R-R, Oh J-H, Cho H, Varshavsky A, Hwang C-S (2014) The N-terminal methionine of cellular proteins as a degradation signal. Cell 156:158-169

Kitamura K, Fujiwara H (2013) The type-2 N-end rule peptide recognition activity of Ubr11 ubiquitin ligase is required for the expression of peptide transporters. FEBS Lett 587:214-219

Kitamura K, Taki M, Tanaka N, Yamashita I (2011) Fission yeast Ubr1 ubiquitin ligase influences the oxidative stress response via degradation of active Pap1 bZIP transcription factor in the nucleus. Mol Microbiol 80:739-755

Kitamura K, Nakase M, Tohda H, Takegawa K (2012) The Ubiquitin ligase Ubr11 is essential for oligopeptide utilization in the fission yeast Schizosaccharomyces pombe. Eukaryot Cell 11:302-310

Kriegenburg F, Jakopec V, Poulsen EG, Nielsen SV, Roguev A, Krogan N, Gordon C, Fleig U, Hartmann-Petersen R (2014) A chaperone-assisted degradation pathway targets kinetochore proteins to ensure genome stability. PLoS Genet 10:e1004140

Kwon YT, Xia Z, Davydov IV, Lecker SH, Varshavsky A (2001) Construction and analysis of mouse strains lacking the ubiquitin ligase UBR1 (E3a) of the N-end rule pathway. Mol Cell Biol 21:8007-8021

Licausi F, Kosmacz M, Weits DA, Giuntoli B, Giorgi FM, Voesenek LA, Perata P, van Dongen JT (2011) Oxygen sensing in plants is mediated by an $\mathrm{N}$-end rule pathway for protein destabilization. Nature 479:419-422
Lu Y, Su C, Unoje O, Liu H (2014) Quorum sensing controls hyphal initiation in Candida albicans through Ubr1-mediated protein degradation. Proc Natl Acad Sci U S A 111:1975-1980

Lupas AN, Koretke KK (2003) Bioinformatic analysis of ClpS, a protein module involved in prokaryotic and eukaryotic protein degradation. J Struct Biol 141:77-83

Matta-Camacho E, Kozlov G, Li FF, Gehring K (2010) Structural basis of substrate recognition and specificity in the N-end rule pathway. Nat Struct Mol Biol 17:1182-1187

Nillegoda NB, Theodoraki MA, Mandal AK, Mayo KJ, Ren HY, Sultana R, Wu K, Johnson J, Cyr DM, Caplan AJ (2010) Ubr1 and Ubr2 function in a quality control pathway for degradation of unfolded cytosolic proteins. Mol Biol Cell 21:2102-2116

Piatkov Kl, Brower CS, Varshavsky A (2012) The N-end rule pathway counteracts cell death by destroying proapoptotic protein fragments. Proc Natl Acad Sci U S A 109:E1839-E1847

Prasad R, Kawaguchi S, Ng DT (2010) A nucleus-based quality control mechanism for cytosolic proteins. Mol Biol Cell 21:2117-2127

Rao H, Uhlmann F, Nasmyth K, Varshavsky A (2001) Degradation of a cohesin subunit by the $\mathrm{N}$-end rule pathway is essential for chromosome stability. Nature 410:955-959

Reiss Y, Kaim D, Hershko A (1988) Specificity of binding of $\mathrm{NH}_{2}$-terminal residue of proteins to ubiquitin-protein ligase. J Biol Chem 263:2693-2698

Ridenour JB, Smith JE, Hirsch RL, Horevaj P, Kim H, Sharma S, Bluhm BH (2013) UBLI of Fusarium verticillioides links the $\mathrm{N}$-end rule pathway to extracellular sensing and plant pathogenesis. Environ Microbiol, doi:10.1111/1462-2920.12333

Román-Hernández G, Grant RA, Sauer RT, Baker TA (2009) Molecular basis of substrate selection by the $\mathrm{N}$-end rule adaptor protein ClpS. Proc Natl Acad Sci U S A 106:8888-8893

Schuenemann VJ, Kralik SM, Albrecht R, Spall SK, Truscott KN, Dougan DA, Zeth K (2009) Structural basis of $\mathrm{N}$-end rule substrate recognition in Escherichia coli by the ClpAP adaptor protein ClpS. EMBO Rep 10:508-514

Stolz A, Besser S, Hottmann H, Wolf DH (2013) Previously unknown role for the ubiquitin ligase Ubr1 in endoplasmic reticulum-associated protein degradation. Proc Natl Acad Sci U S A 110:15271-15276

Summers DW, Wolfe KJ, Ren HY, Cyr DM (2013) The Type II Hsp40 Sis1 cooperates with $\mathrm{Hsp} 70$ and the E3 ligase Ubr1 to promote degradation of terminally misfolded cytosolic protein. PLoS One 8:e52099

Tasaki T, Zakrzewska A, Dudgeon DD, Jiang Y, Lazo JS, Kwon YT (2009) The substrate recognition domains of the $\mathrm{N}$-end rule pathway. J Biol Chem 284:1884-1895

Tasaki T, Sriram SM, Park KS, Kwon YT (2012) The N-end rule pathway. Annu Rev Biochem 81:261-289

Theodoraki MA, Nillegoda NB, Saini J, Caplan AJ (2012) A network of ubiquitin ligases is important for the dynamics of misfolded protein aggregates in yeast. J Biol Chem 287:23911-23922

Turner GC, Du F, Varshavsky A (2000) Peptides accelerate their uptake by activating a ubiquitin-dependent proteolytic pathway. Nature 405:579-583

Varshavsky A (2011) The N-end rule pathway and regulation by proteolysis. Protein Sci 20:1298-1345

Wang KH, Roman-Hernandez G, Grant RA, Sauer RT, Baker TA (2008) The molecular basis of $\mathrm{N}$-end rule recognition. Mol Cell 32:406-414

Xia Z, Turner GC, Hwang C-S, Varshavsky A (2008a) Amino acids induce peptide uptake via accelerated degradation of CUP9, the transcriptional repressor of the PTR2 peptide transporter. J Biol Chem 283:28958-28968

Xia Z, Webster A, Du F, Piatkov K, Ghislain M, Varshavsky A (2008b) Substrate-binding sites of UBR1, the ubiquitin ligase of the $\mathrm{N}$-end rule pathway. J Biol Chem 283:24011-24028

Xu Z, Payoe R, Fahlman RP (2012) The C-terminal proteolytic fragment of the breast cancer susceptibility type 1 protein (BRCA1) is degraded by the $\mathrm{N}$-end rule pathway. J Biol Chem 287:7495-7502

Yamamoto K, Youle RJ (2013) PINK1 is degraded through the N-end rule pathway. Autophagy 9:1758-1769

doi:10.1186/2193-1801-3-257

Cite this article as: Kitamura: The ClpS-like N-domain is essential for the functioning of Ubr11, an N-recognin in Schizosaccharomyces pombe. SpringerPlus 2014 3:257. 